\title{
Gain-Scheduling Control Solutions for Magnetic Levitation Systems
}

\section{Claudia-Adina Bojan-Dragos, Mircea-Bogdan Radac, Radu-Emil Precup, Elena-Lorena Hedrea, Oana-Maria Tănăsoiu}

Department of Automation and Applied Informatics, Politehnica University of Timisoara, Bd. V. Parvan 2, 300223 Timisoara, Romania E-mail: claudia.dragos@upt.ro,mircea.radac@upt.ro,radu.precup@upt.ro, elena.constantin@student.upt.ro, oana.tanasoiu@student.upt.ro

\footnotetext{
Abstract: The paper presents three Gain-Scheduling Control (GS-C) design procedures starting with classical Proportional-Integral (PI) controllers, resulting in PI-GS-C structures for positioning control of a Magnetic Levitation System (MLS) with two laboratory electromagnets. The nonlinear mathematical model of the MLS is first linearized at seven operating points and next stabilized by a state feedback control structure. Three PI-GS-C structures, namely as Lagrange, Cauchy and Switching GS versions, are next designed in order to ensure zero steady-state control error and the switching between PI controllers. All control solutions are validated by real-time experiments.
}

Keywords: Gain-Scheduling Control; magnetic levitation systems; Proportional-Integral control; real-time experiments

\section{Introduction}

One of the topics of Cognitive Info-Communications is dealing with Cognitive Control [1-5]. Cognitive Control is defined as [6]: "Cognitive control theory is an interdisciplinary branch of engineering, mathematics, informatics, control theory and the cognitive/social sciences. It deals with the dynamics of individual and/or collective cognitive phenomena. The theories and methodologies of Cognitive Control give control theoretical interpretations of such dynamics in order to explain and control cognitive phenomena, as well as to apply them in system control design, without necessary distinguishing between biological and artificial aspects." 
The paper focuses on a Magnetic Levitation System with a Two Electromagnets (MLS) positioning problem that is a kind of general description of a special type of object balancing. The paper would like to introduce MLS to this multidisciplinary cognitive control field to find synergies between this generic description and various different models emerging in kinematics and various cognitive control methods of humans. The MLS model includes complex natural behaviours that seem to be close to a wide class of balancing process in cognitive control. The opposite way is also important, when cognitive models are used in the control design of such balancing and positioning problems [7]. The paper proposes solutions to the MLS positioning problem in a way that they are general enough to apply for a wider class of the variations of MLS to have better matching to cognitive control.

Several classical and modern control solutions have been proposed to solve the MLS positioning problem including the recent ones: Proportional-Integral (PI)based solutions are presented in [8-11], fuzzy and adaptive control solutions are given in [12-14] with rather general applicability and comparisons, and predictive control solutions are reported in [15-17].

Due to the fact that the linear controllers can work only in some neighbourhood of a single operating point, the Gain-Scheduling (GS) technique is one of the most common used controller design approaches for nonlinear systems. GS is popular nowadays in many engineering applications because the scheduling variable should "vary slowly" and "capture the plant's nonlinearities" [18-20]. Some of the current approaches to GS are pointed out as follows: an analysis of two and three types of GS control (as Linear Parameter-Varying (LPV) plant scheduling on exogenous parameters, LPV plant scheduling on reference trajectory and LPV plant scheduling on plant output) for nonlinear systems and the conditions which guarantee the stability, robustness and performance of the overall gain-scheduled design are given in [19] and [20]; two GS control design procedures, which are supported by Lyapunov's stability theory, are suggested in [21], they guarantee parameter dependent quadratic stability at a certain cost; fuzzy-based GS of exact feed-forward linearization control and sliding mode controllers for magnetic ball levitation system are proposed in [13]. A high gain adaptive output feedback control to a magnetic levitation system is discussed in [22]. A ProportionalIntegral Gain-Scheduling Control (PI-GS-C) system for second-order LPV systems, which excludes time varying delay and uses a Smith predictor, is given in [23]. Assuming an equilibrium manifold linearization model, a GS control method for nonlinear shock motion is proposed in [24]. A GS controller is designed in [18] on the basis of an LPV system using Lyapunov's stability theory. GS deals in [25] and [26] with the adaptation of gains of a robust evolving cloud-based controller (RECCo) designed for a class of nonlinear processes; the robust modification of the adaptive laws and the performance analysis are introduced. A practical implementation of RECCo with normalized data space for a heatexchanger plant is reported in [27]. Other interesting adaptive GS control techniques for real practical applications are given in [28-31]. 
This paper treats the design and real-time validation of the following control solutions which are able to carry out the position control of the magnetic sphere that belongs to MLS [32]. First of all a state feedback control solution and a control solution (CS) based on PI controllers are designed for each operating point in order to stabilize and to ensure the zero steady-state control error by applying the control signal only to the top electromagnet. The control signal applied to the bottom electromagnet is neglected because it is considered also as an exogenous disturbance. The new contribution of this paper with respect to the state-of-the-art is the real-time application of three GS controllers to MLSs. The first GS version is based on a generalization from the monovariable case to the multivariable one of the Lagrange interpolating parameter value method, the second GS version is based on a Cauchy kernel distance metric, and the third GS version is based on the switching between PI linear controllers. A comparative analysis of the proposed GS versions developed for stabilized Magnetic Levitation System (sMLS) is given to highlight the achieving of the specified control system performance.

The GS controllers proposed in this paper are important because although the conclusions cannot be generalized, they are general and applicable too many processes. These process applications include large-scale systems [33], multi-tank systems [34], fuzzy modelling [35-39], robotics [40-43], fuzzy control [44, 45], motion control [46-48], software agents [49], discrete-event systems [50, 51].

The paper is organized as follows: Section 2 gives the mathematical models of the sMLS. Three case studies corresponding to the GS-C solutions - namely Lagrange, Cauchy and Switching GS - are presented in Section 3. The experimental results and the control performance are presented in Section 4. The conclusions are highlighted in Section 5.

\section{Mathematical Models of Magnetic Levitation System}

The controlled plant taken into consideration in this paper is the complete control laboratory system built around the MLS. The MLS laboratory equipment includes both hardware and software components: two electromagnets (EM1 - the upper electromagnet and EM2 - the lower electromagnet), the ferromagnetic sphere, sensors to detect the position of the sphere, computer interface, drivers, power supply unit, connection cables and an acquisition board of type RT-DAC4 / PCI. When both electromagnets (EM1 and EM2) are used, the control signal applied to EM2 can be used as an additional force leading to multivariable control systems. This feature is also useful in robust applications. Moreover, EM2 can be considered as a cause of disturbance inputs that act as external force excitations. 
The schematic diagram of the MLS laboratory setup is presented in Figure 1, where $m$ is the mass of the sphere, $F_{e m 1}$ and $F_{e m 2}$ are the electromagnetic forces, and $F_{g}$ is the gravity force [32].

The nonlinear state-space mathematical model of MLS is [32]:

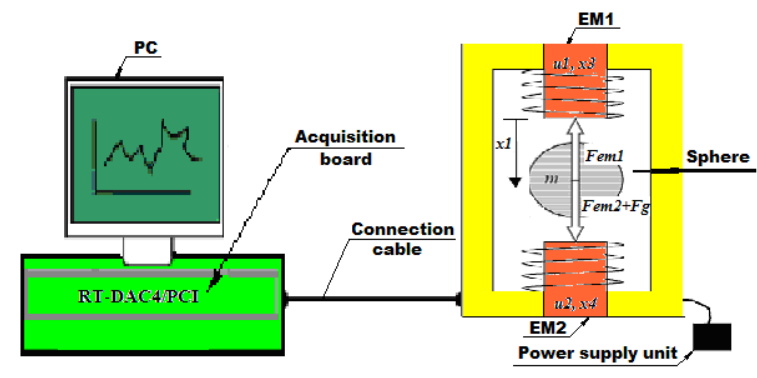

Figure 1

The MLS laboratory setup

$$
\begin{aligned}
\dot{x}_{1}(t)= & v(t), \\
\dot{v}(t)=- & \frac{i_{E M 1}^{2}(t) \cdot F_{e m P 1} \cdot \exp \left(-x_{1}(t) / F_{e m P 2}\right)}{m \cdot F_{e m P 2}}+g \\
& +\frac{i_{E M 2}^{2}(t) \cdot F_{e m P 1} \cdot \exp \left(-\left(x_{d}-x_{1}(t)\right)\right) / F_{e m P 2}}{m \cdot F_{e m P 2}}, \\
\dot{i}_{E M 1}(t)= & \frac{k_{i} \cdot u_{E M 1}(t)+c_{i}-i_{E M 1}(t)}{\frac{f_{i P 1}}{f_{i P 2}} \cdot \exp \left(-x_{1}(t) / f_{i P 2}\right)}, \\
\dot{i}_{E M 2}(t)= & \frac{k_{i} \cdot u_{E M 2}(t)+c_{i}-i_{E M 2}(t)}{f_{i P 1} \cdot \exp \left(-\left(x_{d}-x_{1}(t) / f_{i P 2}\right)\right)} \\
f_{i P 2} & k_{m} \cdot x_{1}(t) .
\end{aligned}
$$

This model corresponds to a strongly unstable fourth-order system, where: $x_{1} \in[0$, $0.0016]$ - the sphere position $(\mathrm{m}), v \in \mathfrak{R}$ - the sphere speed $(\mathrm{m} / \mathrm{s}), i_{\mathrm{EM} 1}$, $i_{\mathrm{EM} 2} \in[0.03884,2.38]$ - the currents in EM1 and EM2, respectively (A), $u_{\mathrm{EM} 1}$, $u_{\mathrm{EM} 2} \in[0.00498,1]$ - the signals applied to EM1 and EM2, respectively (V), and $y$ - the process (plant) output (m). The MLS plant includes both actuators and sensors.

The numerical values of the process parameters are determined analytically and experimentally [32] and get the following values: $D_{s}=0.06$ is the diameter of the sphere, $x_{d}=0.09[\mathrm{~m}]$ is the distance between electromagnets minus sphere diameter, $g=9.81\left[\mathrm{~m} / \mathrm{s}^{2}\right]$ is the gravity acceleration, $m=0.0571[\mathrm{~kg}]$ is the sphere mass, the parameters $k_{i}=0.0243[\mathrm{~A}]$ and $c_{i}=2.5165$ [A] correspond the actuator 
dynamic analysis, $F_{e m P 1}=1.7521 \cdot 10^{-2}[\mathrm{H}], F_{e m P 2}=5.8231 \cdot 10^{-3}[\mathrm{~m}], f_{i P 1}=1.4142 \cdot 10^{-4}$ $[\mathrm{ms}], f_{i P 2}=4.5626 \cdot 10^{-3}[\mathrm{~m}]$.

The nonlinear fourth-order system (1) is reduced to a third-order system

$$
\begin{aligned}
& \dot{x}_{1}(t)=v(t), \\
& \dot{v}(t)=-\frac{i_{E M 1}^{2}(t) \cdot F_{e m P 1} \cdot \exp \left(-x_{1}(t) / F_{e m P 2}\right)}{m \cdot F_{e m P 2}}+g, \\
& \dot{i}_{E M 1}(t)=\frac{k_{i} \cdot u_{E M 1}(t)+c_{i}-i_{E M 1}(t)}{\frac{f_{i P 1}}{f_{i P 2}} \cdot \exp \left(-x_{1}(t) / f_{i P 2}\right)}, \\
& y(t)=k_{m} \cdot x_{1}(t) .
\end{aligned}
$$

with the following state variables: the position $x_{1}$, the sphere speed $v$ and the current $i_{E M 1}$ in EM1 in terms of neglecting EM2. The signals $i_{\mathrm{EM} 2}=0.039$ and $u_{\mathrm{EM} 2}=0.005$ create disturbances.

The characteristics of the sphere position sensor and of the coil current are shown in Figure 2 (a) and (b), respectively. To build the above characteristics it is necessary to measure the position and the current of the electromagnet coil. The electromagnetic \{force $\Leftrightarrow$ position \} and \{force $\Leftrightarrow$ coil current d diagrams are illustrated in Figure 3 (a) and (b), respectively.

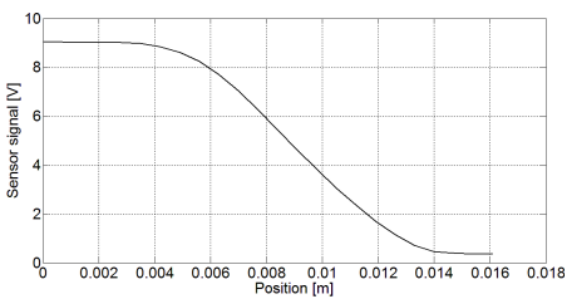

(a)

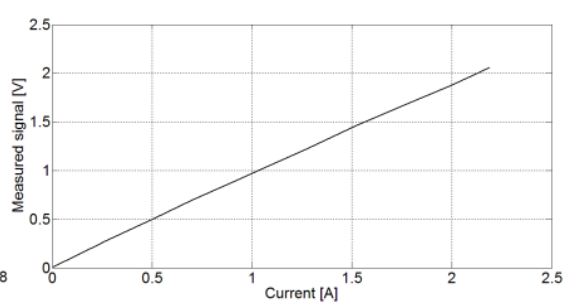

(b)

Figure 2

Characteristics of sphere position sensor (a); characteristics of coil current sensor (b)

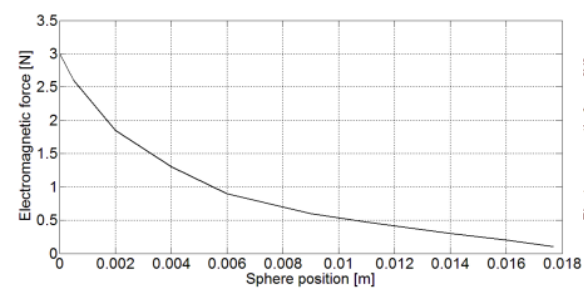

(a)

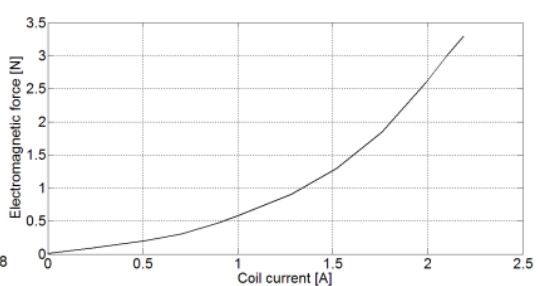

(b)

Figure 3

Electromagnetic force vs. position diagram (a); electromagnetic force vs. coil current diagram (b) 
Taking into account the particularity of the nonlinearities (continuity and monotony), the structural properties of the process are checked with reference to the state-space mathematical model (2) linearized at seven operating points (o.p.s). The following state-space linearized mathematical models (LMMs) are obtained:

$$
\begin{aligned}
& \left\{\begin{array}{l}
\Delta \dot{\mathbf{x}}^{(j)}=\mathbf{A}^{(j)} \Delta \mathbf{x}^{(j)}+\mathbf{b}_{u E M 1}^{(j)} \Delta u_{E M 1}^{(j)}, \\
\Delta y^{(j)}=\mathbf{c}^{T^{(j)}} \Delta \mathbf{x}^{(j)}
\end{array}\right. \\
& \Delta \mathbf{x}^{(j)}=\left[\begin{array}{lll}
\Delta x_{1}^{(j)} & \Delta v^{(j)} & \Delta i_{E M 1}^{(j)}
\end{array}\right]^{T}, \\
& \Delta y^{(j)}=\Delta x_{1}^{(j)}, \\
& \mathbf{A}^{(j)}=\left[\begin{array}{ccc}
0 & 1 & 0 \\
a_{21}^{(j)} & 0 & a_{23}^{(j)} \\
a_{31}^{(j)} & 0 & a_{33}^{(j)}
\end{array}\right], \mathbf{b}_{u E M 1}^{(j)}=\left[\begin{array}{c}
0 \\
0 \\
b_{31}^{(j)}
\end{array}\right], \mathbf{c}^{T^{(j)}}=\left[\begin{array}{lll}
1 & 0 & 0
\end{array}\right] . \\
& \mathbf{A}^{(j)} \in \mathfrak{R}^{3 x 3}, \mathbf{b}_{u E M 1}^{(j)} \in \mathfrak{R}^{3 x 1}, \mathbf{c}^{T^{(j)}} \in \mathfrak{R}^{1 x 3}, \Delta \mathbf{x}^{(j)} \in \mathfrak{R}^{3 x 1}, \Delta u_{E M 1}^{(j)} \in \mathfrak{R},
\end{aligned}
$$

with the matrix parameters

$$
\begin{aligned}
& a_{21}^{(j)}=\frac{i_{E M 10}^{2}}{m} \frac{F_{e m P 1}}{F_{e m P 2}^{2}} e^{-\frac{x_{10}}{F_{e m P 2}}}, a_{23}^{(j)}=-\frac{2 i_{E M 10}}{m} \frac{F_{e m P 1}}{F_{e m P 2}} e^{-\frac{x_{10}}{F_{e m P 2}}}, \\
& a_{31}^{(j)}=-\left(k_{i} u_{E M 10}+c_{i}-i_{E M 10}\right) \frac{x_{10}}{f_{i P 1}} \cdot e^{\frac{x_{10}}{f_{i P 2}}}, a_{33}^{(j)}=-\frac{f_{i P 2}}{f_{i P 1}} \cdot e^{\frac{x_{10}}{f_{i P 2}}}, b_{31}^{(j)}=k_{i} \cdot \frac{f_{i P 2}}{f_{i P 1}} \cdot e^{\frac{x_{10}}{f_{i P 2}}},
\end{aligned}
$$

where $j=\overline{1,7}$ is the index of the operating point $P^{(j)}=\left(x_{1}^{(j)}, v^{(j)}, i_{E M 1}{ }^{(j)}, u_{E M 1}^{(j)}\right)^{T}$ detailed in Table 1. The operating points were chosen from the middle steady-state zone of the sphere position sensor characteristics shown in Figure 2 (a) as it is advised to avoid choosing operating points from the characteristics's extremities, due to instabilities that may occur. The variables in (3) are: $\Delta x_{1}^{(j)}=x_{1}^{(j)}-x_{10}^{(j)}$, $\Delta v^{(j)}=v^{(j)}-v_{0}^{(j)}, \Delta i_{E M 1}^{(j)}=i_{E M 1}^{(j)}-i_{E M 10}^{(j)}, \Delta u_{E M 1}^{(j)}=u_{E M 1}^{(j)}-u_{E M 10}^{(j)}, \Delta y^{(j)}=y^{(j)}-y_{0}^{(j)}$, $j=\overline{1,7}$, representing the differences of the variables $x_{1}^{(j)}, v^{(j)}, i_{E M 1}^{(j)}, u_{E M 1}^{(j)}$ and $y^{(j)}$ with respect to their values at the current operating point $P^{(j)}$, and referred to as $x_{10}^{(j)}, v_{0}^{(j)}, i_{E M 10}^{(j)}, u_{E M 10}^{(j)}$ and $y_{0}^{(j)}$, respectively. The operating points $P^{(j)}$ are considered as state vectors.

The transfer function (t.f) corresponding to the state-space LMMs (3) has the general expression

$$
H_{s M L S}^{(j)}(s)=\mathbf{c}^{T^{(j)}}\left(s \mathbf{I}-\mathbf{A}^{(j)}\right)^{-1} \underline{\mathbf{b}}_{u E M 1}^{(j)}=\frac{k_{P}^{(j)} / \prod_{\eta=1,3} p_{\eta}^{(j)}}{\prod_{\eta=1,3}\left(s-p_{\eta}^{(j)}\right)}=\frac{k_{P C}^{(j)}}{\prod_{\eta=1,3}\left(1+T_{\eta}^{(j)} s\right)},
$$


where $k_{P C}^{(j)}=k_{P}^{(j)} / \prod_{\eta=1,3} p_{\eta}^{(j)}, j=\overline{1,7}, \mathbf{I}$ is the third-order identity matrix and the time constants of the plant are $T_{\eta}^{(j)}=-1 / p_{\eta}^{(j)}, \eta=\overline{1,3}, j=\overline{1,7}$. The plant poles $p_{\eta}^{(j)}, \eta=\overline{1,3}, j=\overline{1,7}$ of the t.f.s. $H_{s M L S}^{(j)}(s)$ at seven operating points are synthesized in Table 1 [52].

Table 1

Operating points and plant poles

\begin{tabular}{|c|c|c|c|c|c|c|c|}
\hline \multirow{2}{*}{$\begin{array}{l}\text { Operating } \\
\text { points } \\
P^{(j)}, \\
j=\overline{1,7}\end{array}$} & \multicolumn{3}{|c|}{ State variables } & \multirow{2}{*}{$\begin{array}{c}\begin{array}{c}\text { Control } \\
\text { signals }\end{array} \\
u_{E M 10}^{(j)} \\
\end{array}$} & \multicolumn{3}{|c|}{$\begin{array}{l}\text { Plant poles } \\
p_{\eta}^{(j)}, \eta=\overline{1,3}, j=\overline{1,7}\end{array}$} \\
\hline & $x_{10}^{(j)}$ & $v_{0}^{(j)}$ & $i_{E M 10}^{(j)}$ & & $p_{1}^{(j)}$ & $p_{2}^{(j)}$ & $p_{3}^{(j)}$ \\
\hline$P^{(1)}$ & 0.0063 & 0 & 1.2185 & 0.48 & 67.49 & -67.49 & -128.34 \\
\hline$P^{(2)}$ & 0.007 & 0 & 1.145 & 0.45 & 59.72 & -59.72 & -149.62 \\
\hline$P^{(3)}$ & 0.0077 & 0 & 1.07 & 0.42 & 52.55 & -52.55 & -174.43 \\
\hline$P^{(4)}$ & 0.0084 & 0 & 1 & 0.39 & 46.25 & -46.25 & -203.36 \\
\hline$P^{(5)}$ & 0.009 & 0 & 0.9345 & 0.36 & 41.05 & -41.05 & -231.94 \\
\hline$P^{(6)}$ & 0.0098 & 0 & 0.89 & 0.34 & 36.5 & -36.52 & -276.39 \\
\hline$P^{(7)}$ & 0.0105 & 0 & 0.83 & 0.32 & 32.06 & -32.06 & -322.22 \\
\hline
\end{tabular}

\section{Control Solutions Design}

\subsection{Design of the State Feedback Control Solution}

The MLS was stabilized using the pole placement method [53] in order to support the development of the proposed control solution. Therefore, the closed-loop system poles $p_{\eta}^{*}=\{-31.81,-41.05,-231.94\}$ were imposed for the linearized models because they can ensure both the stability of the linearized plant and the appropriate state feedback gain matrix to move and keep the sphere at the desired position with respect to EM1. Each set of parameters $\mathbf{k}_{c}^{T(j)}, j=\overline{1,7}$ was tested on the laboratory setup and the best case was obtained for the state feedback gain matrix $\quad \mathbf{k}_{c}^{T}=\mathbf{k}_{c}^{T^{(5)}}=\left[\begin{array}{lll}66.63 & 1.62 & -0.15\end{array}\right] \quad$ (corresponding to the operating point $\left.P^{(5)}\right)$. The performance indices are not acceptable. 
The obtained state feedback gain matrix $\mathbf{k}_{c}^{T}$ was next applied to the LMMs (3) and the following state-space model of the sMLS resulted:

$$
\begin{aligned}
& \Delta \dot{\mathbf{x}}^{(j)}=\mathbf{A}_{x}^{(j)} \Delta \mathbf{x}^{(j)}+\mathbf{b}_{1 x}^{(j)} \Delta u_{1 x}^{(j)}, \\
& \Delta y^{(j)}=\mathbf{c}^{T^{(j)}} \Delta \mathbf{x}^{(j)}, \\
& \Delta \mathbf{x}^{(j)}=\left[\begin{array}{lll}
\Delta x_{1}^{(j)} & \Delta v^{(j)} & \Delta i_{E M 1}^{(j)}
\end{array}\right]^{T}, \\
& \mathbf{A}_{x}^{(j)}=\left[\begin{array}{ccc}
0 & 1 & 0 \\
a_{21}^{(j)} & 0 & a_{23}^{(j)} \\
a_{31}^{(j)} & a_{32}^{(j)} & a_{33}^{(j)}
\end{array}\right], \mathbf{b}_{1 x}^{(j)}=\left[\begin{array}{c}
0 \\
0 \\
b_{31}^{(j)}
\end{array}\right], \mathbf{c}^{T^{(j)}}=\left[\begin{array}{lll}
1 & 0 & 0
\end{array}\right], \\
& \mathbf{A}_{x}^{(j)} \in \mathfrak{R}^{3 x 3}, \mathbf{b}_{1 x}^{(j)} \in \mathfrak{R}^{3 x 1}, \mathbf{c}^{T^{(j)}} \in \mathfrak{R}^{1 x 3}, \Delta u_{1 x}^{(j)} \in \mathfrak{R}, \Delta \mathbf{x}^{(j)} \in \mathfrak{R}^{3 x 1},
\end{aligned}
$$

where the elements of the matrices $\mathbf{A}_{x}^{(j)}$ and $\mathbf{b}_{1 x}^{(j)}$ are

$$
\begin{aligned}
& a_{21}^{(j)}=\frac{i_{E M 1}^{(j)}{ }^{2}}{m} \frac{F_{e m P 1}}{F_{e m P 2}^{2}} e^{-\frac{x_{1}^{(j)}}{F_{e m P 2}}}, a_{23}^{(j)}=-\frac{2 i_{E M 1}^{(j)}}{m} \frac{F_{e m P 1}}{F_{e m P 2}} e^{-\frac{x_{1}^{(j)}}{F_{e m P 2}}}, \\
& a_{31}^{(j)}=-\left(k_{i} u_{E M 1}^{(j)}+c_{i}-i_{E M 1}^{(j)}\right) \frac{x_{1}^{(j)}}{f_{i P 1}} \cdot e^{\frac{x_{1}^{(j)}}{f_{i P 2}}}+66.33 \cdot k_{i} \cdot \frac{f_{i P 2}}{f_{i P 1}} \cdot e^{\frac{x_{1}^{(j)}}{f_{i P 2}}}, \\
& a_{32}^{(j)}=1.62 \cdot k_{i} \cdot \frac{f_{i P 2}}{f_{i P 1}} \cdot e^{\frac{x_{1}^{(j)}}{f_{i P 2}}}, a_{33}^{(j)}=-\frac{f_{i P 2}}{f_{i P 1}} \cdot e^{\frac{x_{1}^{(j)}}{f_{i P 2}}}-0.15 \cdot k_{i} \cdot \frac{f_{i P 2}}{f_{i P 1}} \cdot e^{\frac{x_{1}^{(j)}}{f_{i P 2}}}, \\
& b_{31}^{(j)}=k_{i} \cdot \frac{f_{i P 2}}{f_{i P 1}} \cdot e^{\frac{x_{1}^{(j)}}{f_{i P 2}}} .
\end{aligned}
$$

Two types of transfer functions (t.f.s) of the sMLS, $H_{s M L S}^{(j)}(s)$, were obtained:

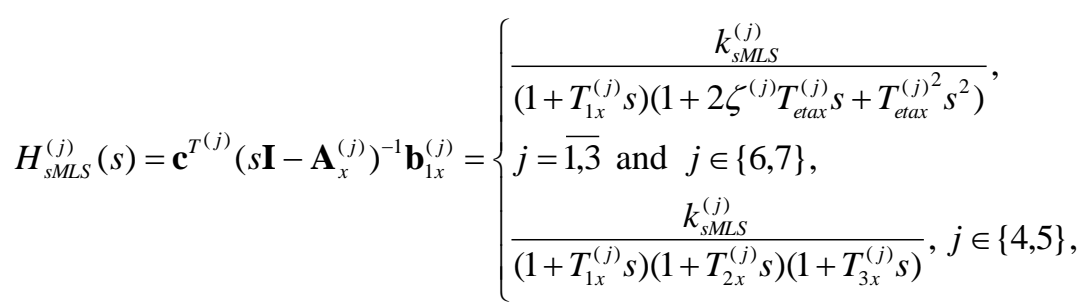

and the parameters are given in Table 2.

\subsection{Design of PI Controllers}

Since the sMLS does not contain an I component, so it could not ensure the zero steady-state control error, the sMLS was included as controlled plant in a cascade control structure (CCS) with PI controller in the outer loop. Seven control 
solutions with PI controllers have been designed using the pole-zero cancellation method [52] depending on the operating points and on the transfer functions (8). The expressions of the t.f.s of the designed PI controllers are rewritten as [52, 5456]:

$H_{P I}^{(j)}(s)=\frac{k_{c}^{(j)}}{s}\left(1+T_{c}^{(j)} s\right)$,

with the PI controller tuning parameters $T_{c}^{(j)}$ and $k_{c}^{(j)}$ :

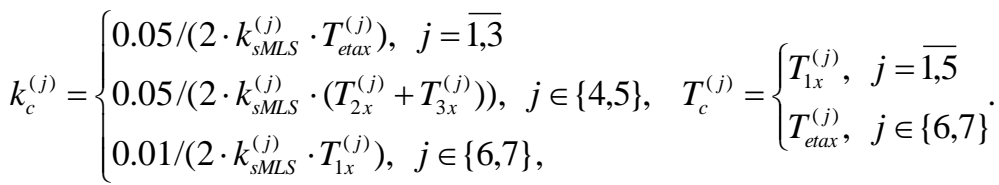

The continuous PI controller (9) is discretized using Tustin's method with the sampling period $T_{s}=0.00025 \mathrm{~s}$. Seven discrete-time PI controllers with the following t.f.s are obtained:

$H_{P I}^{(j)}\left(z^{-1}\right)=\frac{q_{0}^{(j)}+q_{1}^{(j)} z^{-1}}{1-z^{-1}}$,

where $z^{-1}$ is the backward shift operator. The numerical values of tuning parameters and the performance indices of the control systems with PI controllers (from the points of view of overshoot and settling time) are presented in Table 2.

\subsection{Gain-Scheduling Control Solutions Design}

After the design of the discrete-time PI controllers (11) for seven operating points, three GS control solutions, namely Lagrange, Cauchy and Switching GS, are developed in order to improve the control system performance:

$u_{1 x}(k)=u_{1 x}(k-1)+q_{0}(k) e(k)+q_{1}(k) e(k-1)$,

where $k$ is the discrete time argument, $e(k)=r(k)-y(k)$ is the control error sequence, $y(k)$ is the process output sequence, $r(k)$ is the reference input sequence, $q_{i}(k), i \in\{0,1\}$ are the discrete-time PI controller tuning parameters extended with a first-order lag filter:

$q_{i}(k)=\beta \cdot q_{i}(k-1)+q_{i, G S}(k)$,

the parameter $\beta \in\{0.1,0.2,0.3,0.4,0.5\}$ controls the transition speed between different controller parameters, and $q_{i, G S}(k)$ are regarded as reference inputs calculated as follows. 
The detailed block diagram of these three GS versions are given in Figure 4 with focus on SMLS.

Table 2

sMLS parameters and PI tuning parameters

\begin{tabular}{|c|c|c|c|c|c|c|c|c|c|c|}
\hline \multirow{2}{*}{$\begin{array}{l}\text { Operating } \\
\text { points } \\
P^{(j)}, \\
\quad j=\overline{1,7}\end{array}$} & \multicolumn{6}{|c|}{ sMLS parameters } & \multicolumn{2}{|c|}{$\begin{array}{l}\text { Continuous } \\
\text { PI-C tuning } \\
\text { parameters }\end{array}$} & \multicolumn{2}{|c|}{$\begin{array}{l}\text { Discrete PI-C } \\
\text { tuning } \\
\text { parameters }\end{array}$} \\
\hline & $k_{s M L S}^{(j)}$ & $T_{1 x}^{(j)}$ & $T_{2 x}^{(j)}$ & $T_{3 x}^{(j)}$ & $\zeta^{(j)}$ & $T_{\text {etax }}^{(j)}$ & $k_{c}^{(j)}$ & $T_{c}^{(j)}$ & $q_{0}^{(j)}$ & $q_{1}^{(j)}$ \\
\hline$P^{(1)}$ & 0.084 & 0.0988 & - & - & 0.6 & 0.0077 & 38.69 & 0.099 & 3.8337 & -3.8240 \\
\hline$P^{(2)}$ & 0.065 & 0.0778 & - & - & 0.7 & 0.0078 & 48.69 & 0.078 & 3.8000 & -3.7878 \\
\hline$P^{(3)}$ & 0.054 & 0.0618 & - & - & 0.9 & 0.0081 & 57.48 & 0.062 & 3.5673 & -3.5529 \\
\hline$P^{(4)}$ & 0.046 & 0.0485 & 0.0123 & 0.0061 & - & - & 29.73 & 0.049 & 1.4491 & -1.4416 \\
\hline$P^{(5)}$ & 0.041 & 0.0314 & 0.0244 & 0.0043 & - & - & 21.55 & 0.031 & 0.6828 & -0.6774 \\
\hline$P^{(6)}$ & 0.038 & 0.0033 & - & - & 0.9 & 0.0308 & 40.99 & 0.031 & 1.2719 & -1.2617 \\
\hline$P^{(7)}$ & 0.034 & 0.0026 & - & - & 0.7 & 0.0332 & 4.43 & 0.033 & 1.8599 & -1.8460 \\
\hline
\end{tabular}

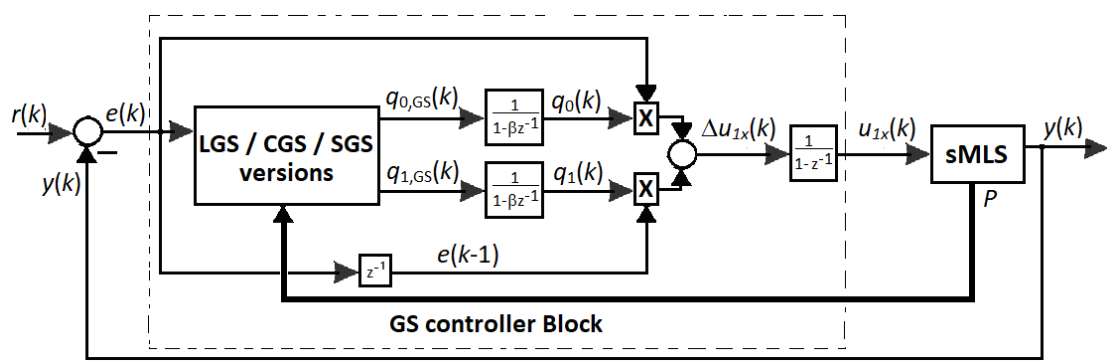

Figure 4

Block diagram of GS versions for the sMLS system

Let $P=\left(x_{1}, v, i_{E M 1}, u_{E M 1}\right)^{T}$ be the current operating point and $\left\|P-P^{(j)}\right\|$ be the Euclidean distance between the current point $P$ and the nearest operating point $P^{(j)}$

The first proposed GS version, namely Lagrange GS version, is based on a generalization of the monovariable case [24] to the multivariable case (the current operating point is in the form of $\left.P=\left(x_{1}, v, i_{E M 1}, u_{E M 1}\right)^{T}\right)$ of the Lagrange interpolating parameter value method:

$q_{i, L G S}=\sum_{j=1}^{n}\left(\frac{\alpha_{L G S}^{(j)}}{\sum_{j=1}^{n} \alpha_{L G S}^{(j)}} \cdot q_{i}^{(j)}\right), \quad i \in\{0,1\}$, 
where

$\alpha_{L G S}^{(j)}=\prod_{l=0, l \neq j}^{n} \frac{\left\|P-P^{(l)}\right\|^{2}}{\left\|P^{(j)}-P^{(l)}\right\|^{2}}$,

the superscripts $j$ denote different operating points, $n=7, L G S$ is Lagrange GS version, and all coefficients $\alpha_{L G S}^{(j)}$ in the first summation in (14) are normalized to add up to 1 .

The second GS version is based on a Cauchy kernel distance metric [25-27] resulting in the Cauchy GS control solution. As shown in (13), this approach directly takes into account all previous data samples:

$q_{i, C G S}=\sum_{j=1}^{n}\left(\frac{\alpha_{C G S}^{(j)}}{\sum_{j=1}^{n} \alpha_{C G S}^{(j)}} \cdot q_{i}^{(j)}\right), \quad i \in\{0,1\}$,

where

$\alpha_{C G S}^{(j)}=\sum_{j=1}^{n} \frac{1}{1+\left\|P-P^{(j)}\right\|^{2}}$,

and $C G S$ is Cauchy GS version.

The third GS version is different to the first two ones as it is based on the switching between PI controllers and the PI controller tuning parameters correspond to the nearest operating point during the real-time experiments. The selection is supported by the Euclidean distance metric resulting in:

$q_{i, S G S}=\sum_{j=1}^{n}\left(\frac{\alpha_{S G S}^{(j)}}{\sum_{j=1}^{n} \alpha_{S G S}^{(j)}} \cdot q_{i}^{(j)}\right), \quad i \in\{0,1\}$,

where

$q_{i, S G S}=q_{i}^{\left(j^{*}\right)}, j^{*}=\arg \min _{j=1, n}\left\|P-P^{(j)}\right\|^{2}, \quad i \in\{0,1\}$,

and $S G S$ is Switching GS version. 


\section{Experimental Results}

All control structures, namely with Lagrange GS, Cauchy GS and Switching GS versions, were tested on the nonlinear laboratory MLS system. Three reference input step-type modifications $\left(R_{1}, R_{2}, R_{3}\right)$ with respect to the EM1 were considered on a testing period of $20 \mathrm{~s}$. The mean squared error $\mathrm{J}_{\mathrm{mse}}$ is computed for all three GS versions

$\mathrm{J}_{\mathrm{mse}}=\frac{1}{N} \sum_{t_{d}=1}^{N}\left(r\left(t_{d}\right)-x_{1}\left(t_{d}\right)\right)^{2}$,

where $x_{1}\left(t_{d}\right)$ is the real position of the sphere at time moment $t_{d}=1 \ldots N$, and $N=80000$ is the number of samples. The performance index $\mathbf{J}_{\text {mse }}$ is measured after carefully experimenting with the controllers in the proposed order \{Lagrange, Cauchy, Switching, Lagrange, Cauchy, Switching, ...\}, to ensure that the timevarying parameters of the equipment uniformly affect all controllers. The boxplot statistics of $J_{\mathrm{mse}}$ over $\beta$ for the Lagrange, Cauchy and Switching GS versions are presented in Figure 5 as the result measured after ten measurements.

A comparative analysis of $J_{m s e}$ over five values of $\beta$ for the designed GS versions, illustrated in Figure 6, highlights that the worst performance is noticed in the Lagrange GS version and the best performance was obtained in the Cauchy GS version in most cases of $\beta$. Moreover, the results indicate that for $\beta=0.3$ the best performance was obtained by the control solution with the third GS version.

Five real-time experimental scenarios were conducted for three step type modifications of the reference input. All results include the evolutions of sphere position $x_{1}(t)$ versus time $t$ for Lagrange, Cauchy and Switching GS control solutions designed for sMLS with $\beta=0.1$ in Figure $6, \beta=0.2$ in Figure 7, $\beta=0.3$ in Figure $8, \beta=0.4$ in Figure 9 , and $\beta=0.5$ in Figure 10.

The following conclusions are drawn by the analysis of the plots given in Figures 6 to 10: (1) The zero steady-state control error is ensured in all versions and also the reference input is well tracked. (2) Due to the nonlinearities of the plant and to the presence of the complex conjugated poles in the cases of the operating points $P^{(1)}-P^{(3)}, P^{(6)}$ and $P^{(7)}$, some oscillations occur at the beginning of transient responses and during the real-time experiments. (3) The proposed control structures design and the obtained results depend on the choice and number of operating points. 

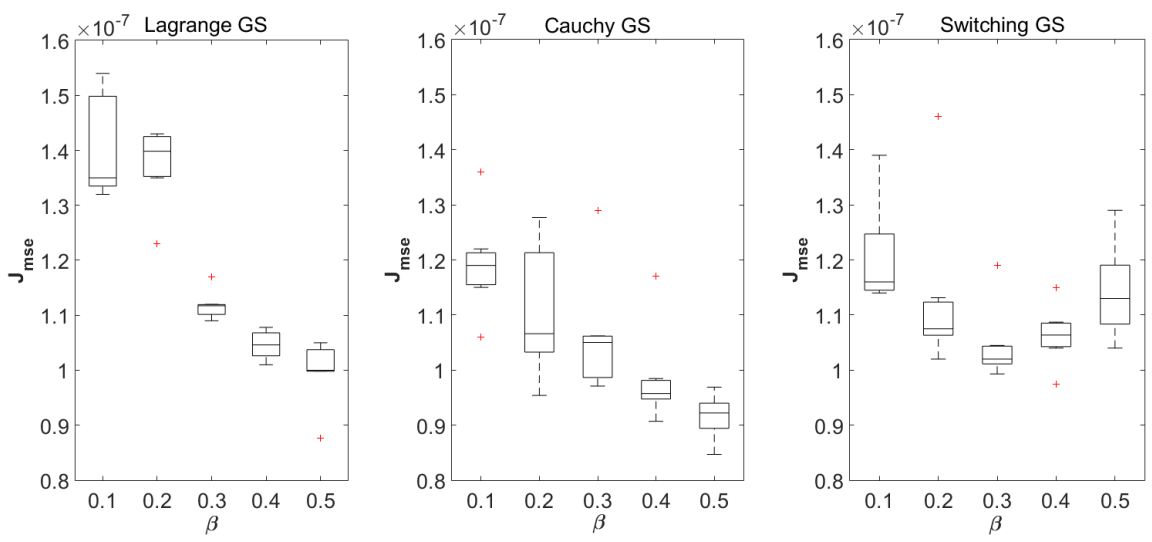

Figure 5

Boxplot statistics of $J_{\mathrm{mse}}$ over $\beta$ for the Lagrange, Cauchy and Switching GS versions, for 10 measurements. Outliers are in red

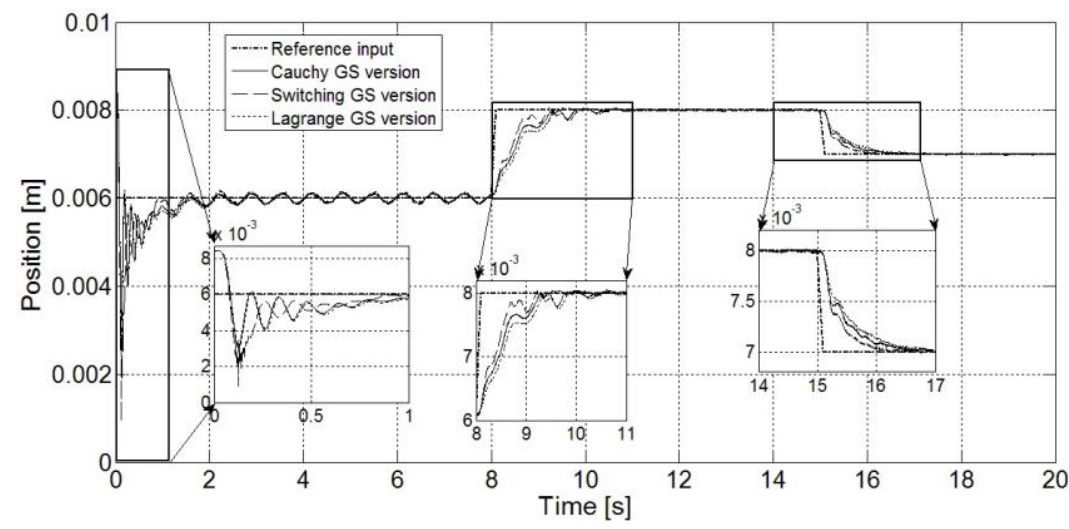

Figure 6

Sphere position $x_{1}(t)$ versus time $t$ for Lagrange, Cauchy and Switching Gain-Scheduling control solutions designed for sMLS with $\beta=0.1$

\section{Conclusions}

The paper has presented the design of three nonlinear gain-scheduling control solutions developed in order to control the position of the sphere in an MLS. All control system structures were tested on the nonlinear model accepting the main values of the parameters given in [32]. Three gain-scheduling control solutions were developed to capture the process nonlinearities and to switch from one PI controller to another one while varying slowly.

The real-time experimental results prove that the GS solutions guarantee the improvement of control system performance in terms of step modifications of the reference input. 


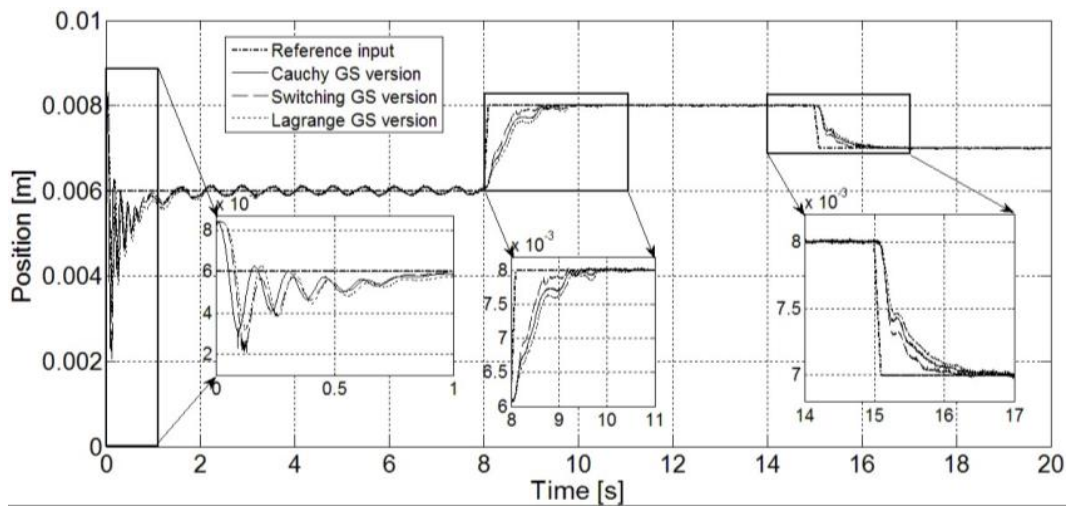

Figure 7

Sphere position $x_{1}(t)$ versus time $t$ for Lagrange, Cauchy and Switching Gain-Scheduling control solutions designed for sMLS with $\beta=0.2$

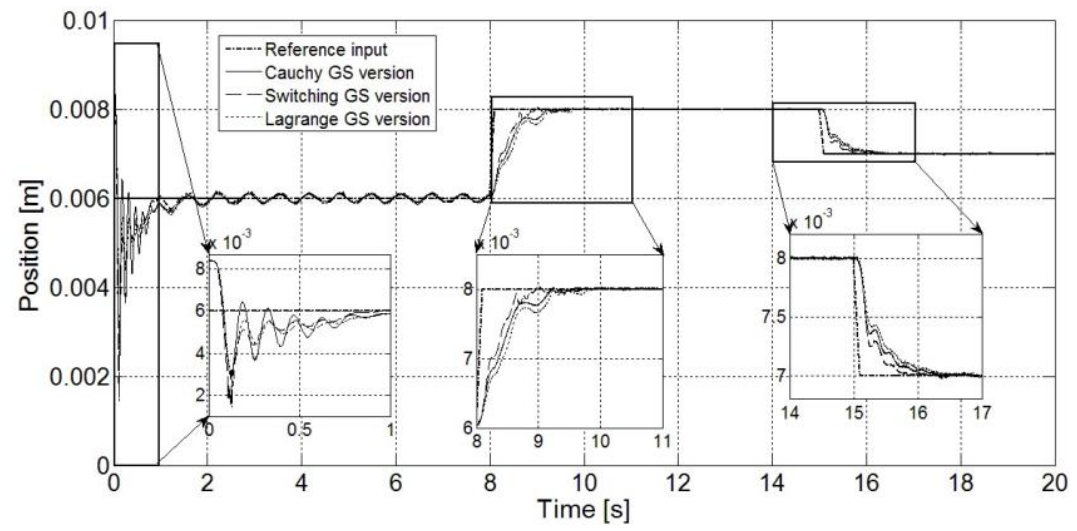

Figure 8

Sphere position $x_{1}(t)$ versus time $t$ for Lagrange, Cauchy and Switching Gain-Scheduling control solutions designed for sMLS with $\beta=0.3$

They ensure zero steady-state control error, small settling times and small overshoots. The values of the mean squared error are small because the order of magnitude of the references input and the controlled output (the sphere position) is millimetres.

Future research will be focused on the design of the control systems with other gain-scheduling control solutions to make comparisons between them, on the design of the control systems with PI(D) fuzzy gain-scheduling controllers, and combined control solutions, which can ensure the improvement of the performance indices. Different modelling and optimization methodologies will be used. 


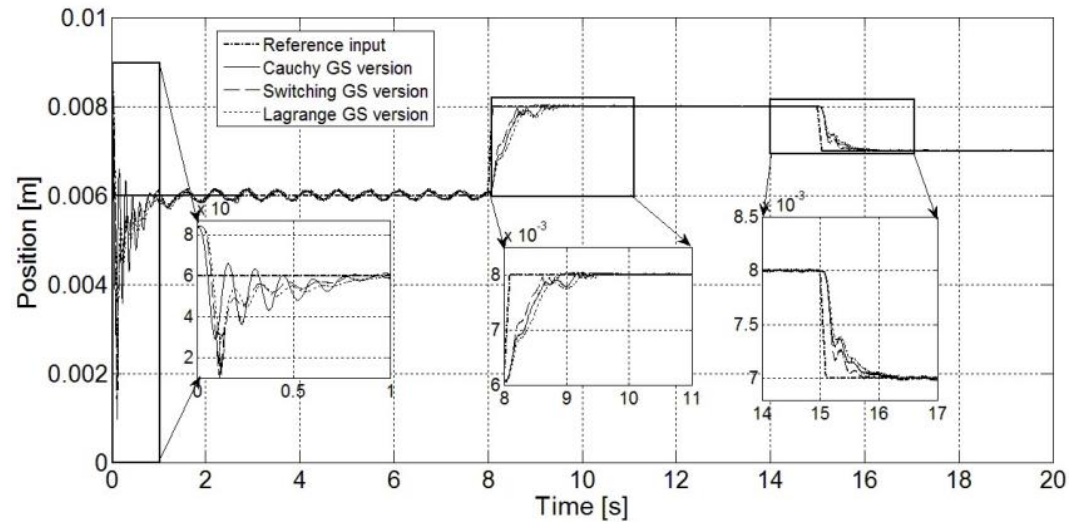

Figure 9

Sphere position $x_{1}(t)$ versus time $t$ for Lagrange, Cauchy and Switching Gain-Scheduling control solutions designed for sMLS with $\beta=0.4$

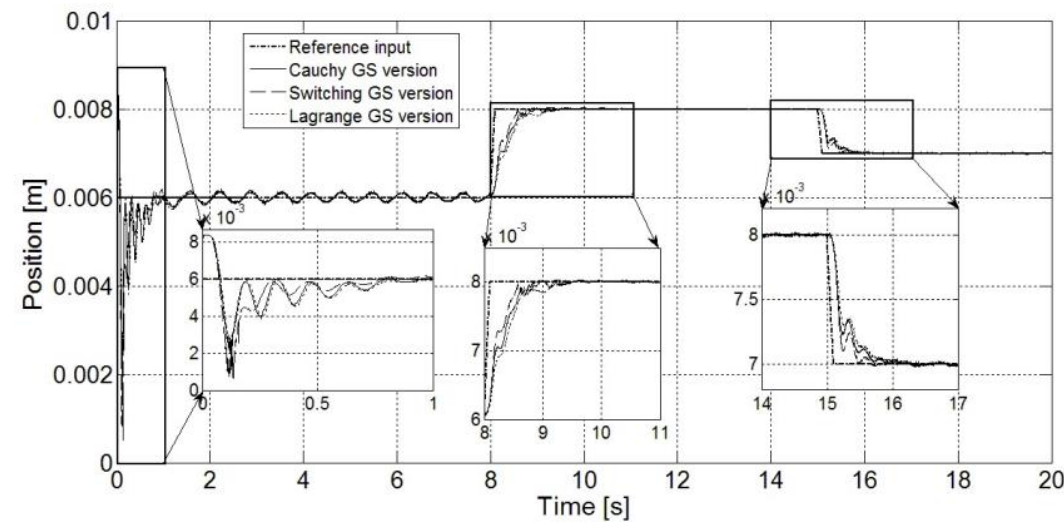

Figure 10

Sphere position $x_{1}(t)$ versus time $t$ for Lagrange, Cauchy and Switching Gain-Scheduling control solutions designed for sMLS with $\beta=0.5$

\section{Acknowledgement}

This work was supported by the research grant PCD-TC-2017 of the Politehnica University of Timisoara, Romania.

\section{References}

[1] P. Baranyi, A. Csapó: Cognitive Infocommunications: CogInfoCom, Proc. $11^{\text {th }}$ IEEE International Symposium on Computational Intelligence and Informatics, Budapest, Hungary, 2010, pp. 141-146 
[2] A. Csapó, P. Baranyi: A Unified Terminology for CogInfoCom Applications, Proc. $2^{\text {nd }}$ International Conference on Cognitive Infocommunications, Budapest, Hungary, 2011, pp. 1-6

[3] P. Baranyi, A. Csapó: Definition and Synergies of Cognitive Infocommunications, Acta Polytechnica Hungarica, Vol. 9, No. 1, 2012, pp. 67-83

[4] P. Baranyi, A. Csapo, G. Sallai: Cognitive Infocommunications (CogInfoCom), Springer International Publishing Switzerland, 2015

[5] F. J. Haugen, M. Hansen, R. Schlanbusch, R. Kristiansen: Cognitive Control of Quadrocopter Using Supervisor, Proc. IEEE $4^{\text {th }}$ International Conference on Cognitive Infocommunications, Budapest, Hungary, 2013, pp. $81-86$

[6] J. K. Tar, I. J. Rudas, K. Kósi, Á. Csapó, P. Baranyi: Cognitive Control Initiative, Proc. $3^{\text {rd }}$ International Conference on Cognitive Infocommunications, Kosice, Slovakia, 2012, pp. 579-584

[7] A. S. Al-Araji: Cognitive Non-linear Controller Design for Magnetic Levitation System, Transactions of the Institute of Measurement and Control, Vol. 38, No. 2, 2016, pp. pp. 215-222

[8] P. Kallakuri, L. H. Keel, S. P. Bhattacharyya: Data Based Design of PID Controllers for a Magnetic Levitation Experiment, Proc. $18^{\text {th }}$ IFAC World Congress, Milano, Italy, 2011, pp. 10231-10236

[9] Sakalli, T. Kumbasar, E. Yesil, H. Hagras: Analysis of the Performances of Type-1, Self-tuning type-1 and Interval Type-2 fuzzy PID controllers on the Magnetic Levitation System, Proc. International Conference on Fuzzy Systems, Beijing, China, 2014, pp. 1859-1866

[10] A. Ghosh, T. R. Krishnan, P. Tejaswy, A. Mandal, J. K. Pradhan, S. Ranasingh: Design and Implementation of a 2-DOF PID Compensation for Magnetic Levitation Systems, ISA Transactions, Vol. 53, 2014, pp. 12161222

[11] S. Yadav, S. K. Verma, S. K. Nagar: Optimized PID Controller for Magnetic Levitation System, Proc. $4^{\text {th }}$ IFAC Conference on Advances in Control and Optimization of Dynamical Systems, Tiruchirappalli, India, 2016, pp. 778-782

[12] E. Shameli, M. B. Khamesee, J. P. Huissoon: Nonlinear Controller Design for a Magnetic Levitation Device, Microsystem Technologies, Vol. 13, 2007, pp. 831-835

[13] M. Lashin, A. T. Elgammal, A. Ramadan, A. A. Abouelsoud, S. F. M. Assal, A. Abo-Ismail: Fuzzy-based Gain Scheduling of Exact Feedforward Linearization Control and SMC for Magnetic Ball Levitation System: A Comparative Study, Proc. International Conference on Automation, Quality and Testing, Robotics, Cluj-Napoca, Romania, 2014, pp. 1-6 
[14] C.-A. Dragos, S. Preitl, R.-E. Precup, R.-G. Bulzan, C. Pozna, J. K. Tar: Takagi-Sugeno Fuzzy Controller for a Magnetic Levitation System Laboratory Equipment, Proc. International Joint Conferences on Computational Cybernetics and Technical Informatics, Timisoara, Romania, 2010, pp. 55-60

[15] B. Wang, G.-P. Liu, D. Rees: Networked Predictive Control of Magnetic Levitation System, Proc. International Conference on Systems, Man and Cybernetics, San Antonio, TX, USA, 2009, pp. 4100-4105

[16] J. Zietkiewicz: Constrained Predictive Control of a Levitation System, Proc. $16^{\text {th }}$ International Conference on Methods and Models in Automation \& Robotics, Miedzyzdroje, Poland, 2011, pp. 278-283

[17] C.-A. Bojan-Dragos, A.-I. Stinean, R.-E. Precup, S. Preitl, E. M. Petriu: Model Predictive Control Solution for Magnetic Levitation Systems, Proc. $20^{\text {th }}$ International Conference on Methods and Models in Automation \& Robotics, Miedzyzdroje, Poland, 2015, pp. 139-144

[18] A. Ilka: Gain-Scheduled Controller Design, Doctoral thesis, Slovak University of Technology in Bratislava, Bratislava, Slovak Republic, 2015

[19] J. S. Shamma: Analysis and Design of Gain-Scheduled Control Systems, $\mathrm{PhD}$ Thesis, Dept. Mechanical Engineering, Massachusetts Institute of Technology, Cambridge, MA, 1988

[20] J. S. Shamma, M. Athans: Analysis of Gain Scheduled Control for Nonlinear Plants, IEEE Transactions on Automatic Control, Vol. 35, No. 8, 1990, pp. 898-907

[21] V. Veselý, A. Ilka: Gain-scheduled PID Controller Design, Journal of Process Control, Vol. 23, No. 8, 2013, pp. 1141-1148

[22] R. Michino, H. Tanaka, I. Mizumoto: Application of High Gain Adaptive Output Feedback Control to a Magnetic Levitation System, Proc. ICROSSICE International Joint Conference, Fukuoka, Japan, 2009, pp. 970-975

[23] V. Puig, Y. Bolea, J. Blesa: Robust Gain-scheduled Smith PID Controllers for Second Order LPV Systems With Time Varying Delay, IFAC Proceedings Volumes, Vol. 45, No. 3, 2012, pp. 199-204

[24] C. Tao, Y. Daren, B. Wen, Y. Yongbin: Gain Scheduling Control of Nonlinear Shock Motion Based on Equilibrium Manifold Linearization Model, Chinese Journal of Aeronautics, Vol. 20. No. 6, 2007, pp. 481-487

[25] P. Angelov, I. Škrjanc, S Blažič: Robust Evolving Cloud-based Controller for a Hydraulic Plant, Proc. 2013 IEEE Conference on Evolving and Adaptive Intelligent Systems, Singapore, 2013, pp. 1-8

[26] G. Andonovski, S. Blažič, P. Angelov, I. Škrjanc: Analysis of Adaptation Law of the Robust Evolving Cloud-based Controller, Proc. 2015 
International Conference on Evolving and Adaptive Intelligent Systems, Douai, France, 2015, pp. 1-7

[27] G. Andonovski, P. Angelov, S. Blažič, I. Škrjanc: A Practical Implementation of Robust Evolving Cloud-based Controller With Normalized Data Space for Heat-exchanger Plant, Applied Soft Computing, Vol. 48, 2016, pp. 29-38

[28] F. D. Bianchi, R. S. Sánchez-Peña, M. Guadayol: Gain Scheduled Control Based on High Fidelity Local Wind Turbine Models, Renewable Energy, Vol. 37, No. 1, 2012, pp. 233-240

[29] A. I. Dounis, P. Kofinas, C. Alafodimos, D. Tseles: Adaptive Fuzzy Gain Scheduling PID Controller for Maximum Power Point Tracking of Photovoltaic System, Renewable Energy, Vol. 60, 2013, pp. 202-214

[30] B. S. J. Costa, P. P. Angelov, L. A. Guedes: Fully Unsupervised Fault Detection and Identification Based on Recursive Density Estimation and Self-evolving Cloud-based Classifier, Neurocomputing, Vol. 150, Part A, 2015, pp. 289-303

[31] Y.-N. Yang, Y. Yan: Attitude Regulation for Unmanned Quadrotors Using Adaptive Fuzzy Gain-Scheduling Sliding Mode Control, Aerospace Science and Technology, Vol. 54, 2016, pp. 208-217

[32] Inteco Ltd., Magnetic Levitation System 2EM (MLS2EM), User's Manual (Laboratory Set), Inteco Ltd., Krakow, Poland, 2008

[33] F. G. Filip: Decision Support and Control for Large-scale Complex Systems, Annual Reviews in Control, Vol. 32, No. 1, 2008, pp. 61-70

[34] R.-E. Precup, M. L. Tomescu, S. Preitl, E. M. Petriu, J. Fodor, C. Pozna: Stability Analysis and Design of a Class of MIMO Fuzzy Control Systems, Journal of Intelligent and Fuzzy Systems, Vol. 25, No. 1, 2013, pp. 145-155

[35] C. Pozna, N. Minculete, R.-E. Precup, L. T. Kóczy, Á. Ballagi: Signatures: Definitions, Operators and Applications to Fuzzy Modeling, Fuzzy Sets and Systems, Vol. 201, 2012, pp. 86-104

[36] R.-E. Precup, M.-C. Sabau, E. M. Petriu: Nature-inspired Optimal Tuning of Input Membership Functions of Takagi-Sugeno-Kang Fuzzy Models for Anti-lock Braking Systems, Applied Soft Computing, Vol. 27, 2015, pp. 575-589

[37] Zs. Cs. Johanyák: A Modified Particle Swarm Optimization Algorithm for the Optimization of a Fuzzy Classification Subsystem in a Series Hybrid Electric Vehicle, Technicki Vjesnik - Technical Gazette, Vol. 24, No. 2, 2017, pp. 295-301

[38] A. Kumar, D. Kumar, S. K. Jarial: A Hybrid Clustering Method Based on Improved Artificial Bee Colony and Fuzzy C-Means Algorithm, 
International Journal of Artificial Intelligence, Vol. 15, No. 2, 2017, pp. 4060

[39] G. Navarro, D. K. Umberger, M. Manic: VD-IT2, Virtual Disk Cloning on Disk Arrays Using a Type-2 Fuzzy Controller, IEEE Transactions on Fuzzy Systems, Vol. 25, No. 6, 2017, pp. 1752-1764

[40] J. Vaščák, K. Hirota: Integrated Decision-Making System for Robot Soccer, Journal of Advanced Computational Intelligence and Intelligent Informatics, Vol. 15, No. 2, 2011, pp. 156-163

[41] Á. Takács, D. Á. Nagy, I. J. Rudas, T. Haidegger: Origins of Surgical Robotics: From Space to the Operating Room, Acta Polytechnica Hungarica, Vol. 13, No. 1, 2016, pp. 13-30

[42] I. J. Rudas, J. Gáti, A. Szakál, K. Némethy: From the Smart Hands to TeleOperations, Acta Polytechnica Hungarica, Vol. 13, No. 1, 2016, pp. 43-60

[43] N. Dučić, Ž. Ćojbašić, R. Radiša, R. Slavković, I. Milićević: CAD/CAM Design and the Genetic Optimization of Feeders for Sand Casting Process, Facta Universitatis, Series: Mechanical Engineering, Vol. 14, No. 2, 2016, pp. 147-158

[44] S. Vrkalovic, T.-A. Teban, I.-D. Borlea: Stable Takagi-Sugeno Fuzzy Control Designed by Optimization, International Journal of Artificial Intelligence, Vol. 15, No. 2, 2017, pp. 17-29

[45] I. Dzitac, F. G. Filip, M.-J. Manolescu: Fuzzy Logic Is Not Fuzzy: Worldrenowned Computer Scientist Lotfi A. Zadeh, International Journal of Computers Communications \& Control, Vol. 12, No. 6, 2017, pp. 748-789

[46] P. Korondi, H. Hashimoto, T. Gajdar, Z. Suto: Optimal Sliding Mode Design for Motion Control, Proceedings of 1996 IEEE International Symposium on Industrial Electronics, Warsaw, Poland, 1996, pp. 277-282

[47] R. M. Del Toro, M. C. Schmittdiel, R. E. Haber-Guerra, R. Haber-Haber: System Identification of the High Performance Drilling Process for Network-Based Control, Proceedings of 2007 ASME International Design Engineering Technical Conferences and Computers and Information in Engineering Conference Las Vegas, NV, USA, 2007, pp. 827-834

[48] R.-E. Precup, R.-C. David, E. M. Petriu: Grey Wolf Optimizer Algorithmbased Tuning of Fuzzy Control Systems with Reduced Parametric Sensitivity, IEEE Transactions on Industrial Electronics, Vol. 64, No. 1, 2017, pp. 527-534

[49] O. Arsene, I. Dumitrache, I. Mihu: Expert System for Medicine Diagnosis Using Software Agents, Expert Systems with Applications, Vol. 42, No. 4, 2015, pp. 1825-1834 
[50] M. Markiewicz, L. Gniewek: Conception of Hierarchical Fuzzy Interpreted PETRI Net, Studies in Informatics and Control, Vol. 26, No. 2, 2017, pp. $151-160$

[51] G.-Y. Zhang, W.-G. Zhao, X.-T. Yan: Collaborative Design Implementation on the PN-PDDP Model for the Complex Coupled Rotor Systems, Control Engineering and Applied Informatics, Vol. 19, No. 3, 2017, pp. 69-78

[52] C.-A. Bojan-Dragos, S. Preitl, R.-E. Precup, S. Hergane, E. G. Hughiet, A.I. Szedlak-Stinean: State Feedback and Proportional-Integral-Derivative Control of a Magnetic Levitation System, Proc. 14 ${ }^{\text {th }}$ International Symposium on Intelligent Systems and Informatics, Subotica, Serbia, 2016, pp. 111-116

[53] R.-E. Precup, S. Preitl: Popov-type Stability Analysis Method for Fuzzy Control Systems, Proc. Fifth European Congress on Intelligent Technologies and Soft Computing, Aachen, Germany, 1997, Vol. 2, pp. 1306-1310

[54] C.-A. Bojan-Dragos, R.-E. Precup, S. Preitl, S. Hergane, E. G. Hughiet, A.I. Szedlak-Stinean: Proportional-Integral Gain-Scheduling Control of a Magnetic Levitation System, Proc. $20^{\text {th }}$ International Conference on System Theory, Control and Computing, Sinaia, Romania, 2016, pp. 1-6

[55] T. Haidegger, L. Kovács, R.-E. Precup, B. Benyó, Z. Benyó, S. Preitl: Simulation and Control for Telerobots in Space Medicine, Acta Astronautica, Vol. 181, No. 1, 2012, pp. 390-402

[56] L. Kovács: A Robust Fixed Point Transformation-Based Approach for Type 1 Diabetes Control, Nonlinear Dynamics, Vol. 89, No. 4, 2017, pp. 2481-2493 